\title{
„Total Digital!“ geht weiter bis 2022! - Nächste offizielle Einreichungsfrist ist der 31. Oktober 2020
}

https://doi.org/10.1515/bd-2020-0066

Der Deutsche Bibliotheksverband e.V. (dbv) wird sein Förderprogramm „Total Digital! Lesen und erzählen mit digitalen Medien“ als Teil des Bundesprogramms „Kultur macht stark. Bündnisse für Bildung“ wie geplant fortsetzen. Das Bundesministerium für Bildung und Forschung (BMBF) hat nach Abschluss der Zwischenbegutachtung entschieden, die Förderung weiterzuführen. Bundesweit können somit Bibliotheken und ihre lokalen Partnereinrichtungen bis 2022 Projekte und Aktionen für drei- bis 18-jährige Kinder und Jugendliche beim dbv beantragen. Aufgrund der aktuellen Corona-bedingten Schutzmaßnahmen beantragen viele Bündnisse statt der Präsenzprojekte vermehrt kontaktlose Projekte mit digitalen Medien.

„Die Schließung von Schulen, Horten und außerschulischen Bildungseinrichtungen bedeutet für Kinder und Jugendliche mit schwierigen Bildungsvoraussetzungen derzeit eine Verschärfung ihrer Situation“, sagt Barbara Schleihagen, Bundesgeschäftsführerin des dbv. „Bibliotheken als wichtige außerschulische Bildungseinrichtungen suchen daher nach Wegen, wie sie Kindern und Jugendlichen auch jetzt und künftig interessante und kostenfreie kulturelle Bildungsangebote unterbreiten können. Gerade diese Zeiten zeigen, wie wichtig Leseförderung, kulturelle Teilhabe und der souveräne Umgang mit Medien ist“, so Schleihagen weiter. „Wir freuen uns daher sehr, dass wir weiterhin Programmpartner des BMBF sein werden.“

Die nächste Ausschreibungsrunde für Projekte im Jahr 2021 endet am 31. Oktober 2020. Bis Mitte September 2020 werden Projektanträge, deren Durchführung noch für den Herbst 2020 geplant sind, auch außer der Reihe entgegengenommen. Detaillierte Informationen sowie Konzepte für kontaktlose Projekte sind auf www.lesen-und-digitale-medien.de zu finden.

\section{„Total Digital!“}

Der dbv beteiligt sich seit 2018 mit „Total Digital!“ am Förderprogramm „Kultur macht stark. Bündnisse für Bildung“ des Bundesministeriums für Bildung und Forschung (BMBF). „Total Digital!“ richtet sich in erster Linie an junge Menschen, die von bildungsbenachteiligenden Risiken betroffen sind. Mit „Kultur 
macht stark“ fördert das BMBF von 2018 bis 2022 außerschulische Projekte der Kulturellen Bildung für mehr gesellschaftliche Teilhabe und Chancengleichheit mit 250 Mio. Euro. Neben dem dbv sind 28 weitere Verbände und Initiativen Programmpartner von „Kultur macht stark“.

\section{Der Deutsche Bibliotheksverband e.V. (dbv)}

Der Deutsche Bibliotheksverband e.V. (dbv) vertritt mit seinen mehr als 2.100 Mitgliedern bundesweit rund 10.000 Bibliotheken mit 25.000 Beschäftigten und 11 Mio. Nutzer^innen. Sein zentrales Anliegen ist es, Bibliotheken zu stärken, damit sie allen Bürger`innen freien Zugang zu Informationen ermöglichen. Der Verband setzt sich ein für die Entwicklung innovativer Bibliotheksleistungen für Wissenschaft und Gesellschaft. Als politische Interessensvertretung unterstützt der dbv die Bibliotheken insbesondere auf den Feldern Informationskompetenz und Medienbildung, Leseförderung und bei der Ermöglichung kultureller und gesellschaftlicher Teilhabe für alle Bürger*innen.

www.bibliotheksverband.de

\section{Kontakte: Deutscher Bibliotheksverband e.V. (dbv)}

Brigitta Wühr, Projektleiterin „Total Digital“

Tel.: 0306 / 449899 1, E-Mail: wuehr@bibliotheksverband.de Kristin Bäßler, Leitung Kommunikation / Pressesprecherin Tel.: 030 / 6449899 25, E-Mail: presse@bibliotheksverband.de URL: www.bibliotheksverband.de 